\title{
ESTRÉS LABORAL Y CALIDAD DE VIDA \\ ASOCIADA CON LA SALUD EN LOS EMPLEADOS \\ DE UNA INSTITUCIÓN DE EDUCACIÓN \\ SUPERIOR DE EL SALVADOR
}

\author{
Gustavo Paniagua \\ Departamento de Psicología \\ Facultad de Ciencias Sociales "Profesor y Doctor Santiago Echegoyén" \\ Universidad Evangélica de El Salvador \\ francisco.paniagua@uees.edu.sv
}

Recibido 22/01/16

Aceptado 27/05/16

\section{Resumen}

El estrés es un mecanismo que se generó a lo largo de la evolución para favorecer la supervivencia de los seres vivos. Este proceso ha estado presente en la especie humana desde sus inicios; sin embargo, la forma en que se vivencia el estrés en la actualidad ha sido asociado con la generación de diversas problemáticas de salud. El presente estudio pretende indagar la relación entre el estrés laboral y la calidad de vida asociada a la salud en el personal permanente de una Institución de Educación Superior del Área Metropolitana de San Salvador. Para llevar a cabo este proyecto, se utilizó un enfoque cuantitativo, con un diseño no experimental, transeccional y correlacional. Se aplicaron a una muestra de 150 participantes dos escalas psicológicas: el Cuestionario sobre el Estado de Salud "SF36 " y el Cuestionario de Estrés Laboral "JSS" para medir la calidad de vida asociada a la salud y el estrés laboral, respectivamente. Los resultados sugieren que ambas variables están relacionadas de una forma inversamente proporcional, adquiriendo diversos matices a medida que se analizaron a la luz de múltiples factores sociodemográficos, lo cual es congruente con investigaciones realizadas por otros autores. Finalmente se realizan recomendaciones sobre nuevas líneas de investigación sobre la temática, así como también sugerencias de intervención para favorecer la disminución del estrés y la buena calidad de vida en la organización.

PALABRAS CLAVE: estrés laboral; calidad de vida; salud; Síndrome de Burnout; universidades; El Salvador. 


\section{STRESS RELATED TO JOBS AND QUALITY OF LIFE ASSOCIATED WITH HEALTH STAFF HIRED FULL-TIME AT AN INSTITUTION OF HIGHER EDUCATION OF EL SALVADOR}

\section{Summary}

Stress is a mechanism that was generated throughout evolution to encourage the survival of living beings. This process has been presented in humans since its inception, in which stress is experienced at present and has been associated with the generation through various health issues. The present study aims to investigate the relationship between the stress of holding a job and the quality of life associated with health in the permanent staff of an institution Higher Education Metropolitan Area of San Salvador. To carry out this project, a quantitative approach, with a non-experimental, correlationtransactional was used; they applied this to a sample of 150 participants, two psychological scales, the questionnaire health "SF-36" and Job Stress Questionnaire "JSS" to measure quality of life associated with health and labor, and stress. The results suggest that both variables are related in an inversely proportional way, acquiring various nuances as analyzed in the light of multiple socio-demographic factors, which are consistent with research by other authors. Finally, they performed recommendations for further research on the subject, as well as intervention suggestions to promote stress reduction and have a good quality of life within the organization.

Keywords: Job Stress; Quality of life associated with health; Burnout Syndrome; Universities; El Salvador.

\section{INTRODUCCIÓN}

El estrés es una respuesta adaptativa milenaria que ha estado presente en la humanidad desde sus orígenes. En sus raíces, es un mecanismo diseñado para la supervivencia de nuestros antepasados en un mundo hostil, lleno de peligros y amenazas. Sin embargo, en la sociedad contemporánea, aquella respuesta que una vez ayudó a los primeros humanos a subsistir, se ha convertido en un factor causante de malestar y deterioro de la salud física y mental de buena parte de la población en todas partes del mundo (Feldman, 2010).

Esto se debe a que la vida actual ha cambiado la manera en que se percibe y se experimenta el estrés. Gracias a la particular configuración de las sociedades modernas, la mayoría de personas se encuentran estresadas de forma crónica $\mathrm{y}$, consecuentemente, los procesos psicobiológicos del estrés están activados de forma permanente, lo que causaría consecuencias nocivas para el organismo, pues éste no está diseñado para soportar los efectos biológicos del estrés por tiempos prolongados (Feldman, 2010; Von Hopffgarten, 2013).

De acuerdo a Von Hopffgarten (2013), estas consecuencias nocivas para la salud pueden deberse a que el estrés prolongado puede afectar negativamente el sistema inmunitario, ya que la exposición 
permanente al cortisol (hormona del estrés) desequilibra los dos sistemas de defensa con los que se protege el organismo: el sistema inmunitario humoral, que se encarga de producir anticuerpos para combatir microorganismos; y el sistema inmunitario celular, responsable de luchar contra virus y células cancerosas. La exposición crónica ante la hormona mencionada provoca, por un lado, que las células $\mathrm{T}_{\mathrm{H}} 1$ de la defensa celular se inhiban, lo que deja al individuo vulnerable ante infecciones virales; por otro, las células $\mathrm{T}_{\mathrm{H}} 2$ de la defensa humoral se sobre activan, lo que facilita la aparición de reacciones alérgicas.

En esta misma línea, diversos autores (Bauer-Delto, 2013; Mossop, 2013; Rosenzweig y Leiman, 1992) han sugerido la influencia del estrés en el desarrollo de diversas enfermedades dermatológicas, neurodegenerativas, trastornos psicológicos e incluso condiciones como el "enanismo psicosocial".

El estrés laboral, entendiéndolo como un conjunto de reacciones emocionales, cognitivas, fisiológicasy del comportamiento del trabajador a ciertos aspectos adversos o nocivos del contenido, el entorno o la organización del trabajo (Chiavenato, 2009), no está exento de generar una amplia gama de problemáticas. El proceso de generación de éste puede explicarse a través de una aplicación del modelo de afrontamiento de Lazarus y Folkman (Díaz, 2011), de la siguiente manera:

El individuo realiza una evaluación primaria que implica una revisión de las demandas laborales, es decir, las características del trabajo que requieren un esfuerzo físico o mental, y en consecuencia, tiene costes en estos dos ámbitos.

Luego, se efectúa una evaluación secundaria que consiste en analizar tanto los recursos personales -habilidades, valores, autoeficacia, locus de control-, como los recursos laborales, que son aquellas características que reducen las demandas laborales o estimulan el crecimiento, el aprendizaje y el logro de metas, como por ejemplo, el apoyo social, tecnología, entre otros.

En base a estas dos evaluaciones, el individuo llega a concluir que la situación a la que se enfrenta es un desafío o reto, el cual puede ser superado con relativa facilidad; o por el contrario, una amenaza que puede llegar a desbordar a la persona, hacerle entrar en estados de ansiedad y disminuir su capacidad de respuesta ante la situación. En este sentido, se puede inferir que entre mayor sea el coste físico y/o mental percibido de una demanda laboral, y menores sean los recursos personales y laborales para afrontar dicha situación, la persona muy probablemente experimentará estrés laboral.

Son muchos los estresores que se pueden encontrar en el ámbito laboral. Los más citados son aquellos relacionados con el ambiente físico, el contenido del trabajo, las relaciones interpersonales, las oportunidades de desarrollo y las características organizacionales (Díaz, 2011; Durán, 2010; Peiró y Rodríguez, 2008); sin embargo, cabe aclarar que estas situaciones no son estresoras en sí mismas, sino que dependen inherentemente de la valoración subjetiva individual.

No obstante, entre mayores fuentes de estrés negativo (distrés) perciba la persona, más probabilidad tiene de sufrir sus efectos negativos, entre los más destacables, la disminución cuantitativa y cualitativa en el desempeño laboral, la posibilidad de padecer el Síndrome de Burnout (Herranz-Bellido, Reig-Ferrer y Cabrero-García, 2006) y el aparecimiento de padecimientos y enfermedades mencionadas en párrafos anteriores, 
lo que llevaría a una disminución de la calidad de vida asociada a la salud, entendiéndola como la percepción que un individuo tiene respecto a su nivel de bienestar general, y cómo éste se ve afectado por desequilibrios en la salud, tanto física como mental (Morrison y Bennett, 2008).

En este sentido, el objetivo general de la presente investigación consiste en evaluar los niveles de estrés que están experimentando los empleados a tiempo completo de una institución de educación superior del AMSS, y evidenciar si éstos tienen relación con la calidad de vida asociada a la salud de los mismos.

Conocer esta información es de suma importancia, pues permitiría establecer un panorama general del fenómeno en la institución y establecer un punto de partida sobre el cual elaborar recomendaciones orientadas a mantener niveles de estrés saludables (euestrés) para los empleados con el fin de optimizar el desempeño laboral y evitar niveles excesivos de estrés que puedan generar impactos negativos en la calidad de vida asociada a la salud de los trabajadores.

Como hipótesis general, se sostiene que a mayor nivel de estrés laboral, los empleados evidenciarán menor calidad de vida asociada a la salud. Asimismo, como hipótesis específicas se supone que: (a) Los empleados contratados a tiempo completo experimentarán, en promedio, un nivel de estrés laboral alto; y (b) éstos reflejarán un nivel de calidad de vida asociada a la salud, tanto física como mental, inferior al promedio del grupo normativo de referencia (normas mexicanas).

\section{MÉTODO}

\section{Enfoque y diseño de la investigación}

La presente investigación se realizó bajo un enfoque cuantitativo, utilizando un

diseño no experimental transeccional y correlacional (Hernández, Fernández y Baptista, 2010).

\section{Participantes}

Se trabajó con una muestra representativa de la población de estudio, constituida por 150 personas $(51.3 \%$ pertenecientes al sexo masculino y $48.7 \%$ al sexo femenino), cuyas edades oscilan entre los 19 y 68 años ( $M=36.9$ años; $D E=11.25$ años), y que cumplían con los siguientes criterios: ser empleado activo de la Institución, estar contratado a tiempo completo y poseer un periodo de laborar en la misma igual o mayor a seis meses continuos.

\section{Instrumentos}

Para la recopilación de datos en es esta investigación, se utilizaron dos escalas para medir las dos variables de interés.

Para evaluar la variable "estrés laboral", se utilizó el Cuestionario sobre estrés laboral" (Job Stress Survey 'JSS'), elaborado por Charles Spielberguer con el objetivo de medir la intensidad del estrés laboral. Esta escala cuenta con 30 ítems agrupados en cuatro subescalas: Presión en el trabajo, Falta de apoyo social, Falta de organización y Falta de realización personal. La puntuación de los reactivos se realiza bajo un formato tipo Likert. Las puntuaciones brutas tienen puntos de corte que permiten clasificar los resultados en diferentes niveles de experimentación de estrés (alto, medio y bajo), así como la intensidad con la que se vivencian los factores que se miden en las subescalas. La adaptación cubana de este cuestionario reporta una confiabilidad satisfactoria de la prueba, con coeficientes $\alpha$ de Cronbach superioes a .60 (Marrero, Rivero, Pastor, Fernández y Vergara, 2011). Asimismo, se realizó una prueba piloto en la Institución para evaluar la confiabilidad de la prueba, obteniendo 
resultados igualmente satisfactorios, con puntuaciones $\alpha$ de Cronbach $=.966$.

En cuando a la variable "Calidad de vida asociada a la salud", se aplicó el Cuestionario sobre el Estado de Salud" (SF-36), que mide el nivel de calidad de vida relacionada con la salud. Este instrumento consta de 36 ítems agrupados en ocho subescalas, las cuales se describen en la siguiente tabla (García-Portilla, Bascarán, Sáiz, Bousoño y Bobes, 2006):

\section{Tabla 1}

\section{Subescalas del cuestionario sobre el estado de salud (SF-36)}

\begin{tabular}{ll}
\multicolumn{1}{c}{ Subescala } & \multicolumn{1}{c}{ Aspecto que evalúa } \\
\hline Funcionamiento Físico (FF) & $\begin{array}{l}\text { Grado en que el estado de salud limita la realización de } \\
\text { actividades. }\end{array}$ \\
\hline Rol Físico (RF) & $\begin{array}{l}\text { Grado en que los problemas de salud interfieren con el } \\
\text { funcionamiento ocupacional. }\end{array}$ \\
\hline Dolor (D) & Grado de dolor y su interferencia en la vida del paciente. \\
\hline Salud General (SG) & Percepción personal del estado de salud. \\
\hline Vitalidad (V) & Nivel de energía del paciente. \\
\hline Funcionamiento Social (FS) & $\begin{array}{l}\text { Grado en que el estado de salud interfiere con la vida } \\
\text { sociofamiliar. }\end{array}$ \\
\hline Rol Emocional (RE) & $\begin{array}{l}\text { Grado en que los problemas emocionales afectan el } \\
\text { funcionamiento ocupacional. }\end{array}$ \\
\hline Salud Mental (SM) & Estado de ánimo del paciente durante el último mes. \\
\hline
\end{tabular}

Las subescalas FF, RF, D y SG se puede agrupar para formar la medida sumaria de Salud Física. De igual manera, las subescalas V, FS, RE y SM pueden combinarse para constituir la medida sumaria de Salud Mental (Rodríguez, Merino y Castro, 2009).

Los ítems se puntúan bajo un formato tipo Likert, y las puntuaciones totales de cada dimensión oscilan entre 0 y 100 . No existen puntos de corte, por lo que a mayor puntuación, mejor calidad de vida asociada a la salud.

Este instrumento ha sido adaptado para poblaciones españolas y latinoamericanas (Alonso,PrietoyAntó,1995; Durán,Gallegos y Salinas, 2004; Zúniga, Carrillo-Jiménez, Fos, Gandek y Medina-Moreno, 1999), y ha obtenido resultados satisfactorios en las pruebas de confiabilidad, con coeficientes $\alpha$ de Cronbach superiores a .60. En esa misma línea, la puntuación $\alpha$ de Cronbach obtenida en la prueba piloto realizada en la institución fue de .896.

\section{Procedimiento}

En primer lugar, se notificó vía correo electrónico a todo el personal universitario sobre la realización del estudio y se solicitaba su colaboración en el mismo. Posteriormente se visitó cada una de las unidades administrativas de la universidad y se explicó a la autoridad correspondiente aspectos esenciales del estudio que se estaba realizando. De igual manera, se solicitó a cada jefe de unidad el número de empleados de la misma que cumplían con los requisitos para ser participante de la investigación. Luego, en cada unidad administrativa se designó una persona responsable a quien se le entregó una hoja de consentimiento informado y un juego 
de cuestionarios para cada empleado de la misma, a modo que las personas pudieran contestarlos en el tiempo que tuvieran disponible para ello. Finalmente, se concertó con cada jefe una fecha para recoger los instrumentos contestados.

\section{RESULTADOS}

En primer lugar, una característica relevante que se indagó fue el porcentaje de personas que, aparte de trabajar en la Universidad, laboran en otras instituciones. El 12\% de los participantes reportó trabajar en otras instituciones, el $86 \%$ mencionó laborar únicamente en la Universidad, mientras que un $2 \%$ no respondió este ítem.

También se preguntó a los participantes la frecuencia con la que llevan trabajo a casa. Cabe resaltar que aproximadamente un cuarto del personal (27.3\%) reportó realizar tareas laborales en su hogar al menos una vez por semana, práctica que no es deseable por sus potenciales efectos nocivos para la salud.

En esta misma línea, se cuestionó al personal sobre la frecuencia con que laboran fuera de su horario de trabajo. En este caso, se destaca que un poco más de un tercio de la muestra encuestada (38.7\%) reportó trabajar fuera de horario al menos una vez a la semana. Esta situación tampoco es deseable desde el punto de vista de la salud del trabajador, tomando en cuenta que la jornada laboral a tiempo completo comprende entre 8 y 10 horas de estancia dentro de la Institución.

En cuanto a la hipótesis general de investigación, se ejecutó una prueba de correlación de Pearson entre los puntajes obtenidos en el cuestionario JSS y el cuestionario SF-36 para determinar la relación existente entre el estrés laboral y la calidad de vida asociada a la salud (ver tabla 2).

Tabla 2

Correlaciones entre los puntajes del cuestionario de estrés laboral (JSS) y los puntajes de las escalas de salud física, salud mental y salud general del cuestionario sobre el estado de salud (SF-36)

\begin{tabular}{|c|c|}
\hline Salud Física & $-.354^{* *}$ \\
\hline Salud Mental & $-.428^{* *}$ \\
\hline Salud Total & $-417^{* *}$ \\
\hline
\end{tabular}

Se relacionó el nivel de estrés con la salud física, la salud mental y la salud general de la muestra encuestada. En los tres casos se obtuvieron correlaciones estadísticamente significativas, indicando una relación negativa moderada entre las variables. Esto quiere decir que en la medida que el puntaje de una variable sube, el puntaje de la otra baja; y viceversa.

Con respecto a la calidad de vida asociada a la salud, se compararon los promedios obtenidos en las escalas de salud física y salud mental delSF-36 con las puntuaciones de una muestra mexicana (grupo normativo de referencia). En ambas medidas, el personal de la Institución obtuvo una puntuación media por encima del promedio del grupo de comparación (salud física: El Salvador=82.01; México=79.7. Salud mental: El Salvador=78.64; México=76.7).

En esta misma línea se realizaron pruebas $\mathrm{T}$ de Student para indagar la existencia de 
diferencias en el puntaje de las escalas de salud en base al sexo. En promedio, en las tres escalas de salud física $(\mathrm{t}=2.51, \mathrm{p}<.05)$, salud mental $(\mathrm{t}=2.35, \mathrm{p}<.05)$ y salud general $(t=2.77, p<.01)$ se encontraron diferencias estadísticamente significativas al comparar hombres y mujeres. En las tres mediciones, los hombres reportan mejor salud que las mujeres (ver figura 1 ).

\section{Figura 1}

Comparación de las puntuaciones medias de salud en base al sexo

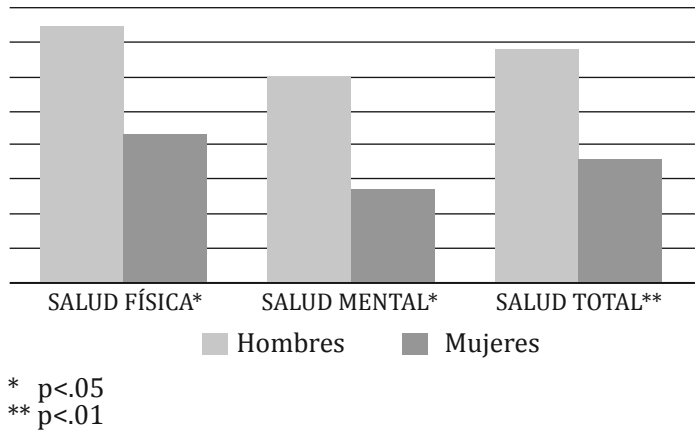

Encuantoalniveldeestréslaboral,elpuntaje promedio del personal institucional fue de 96.63, lo que se interpreta como un nivel de estrés intermedio. Es importante destacar que los participantes se distribuyeron equitativamente entre los diferentes niveles de estrés: un 33.1\% de los encuestados reportó niveles de estrés bajo (puntajes entre 0 y 63.69); el 33.1\% niveles de estrés intermedio (puntajes entre 63.7 y 119.46); y el 33.8\% manifestó niveles de estrés alto (puntajes de 119.47 a 279).

En esta variable también se ejecutó una prueba $\mathrm{T}$ de Student para explorar diferencias en el nivel de estrés en base al sexo. Los resultados evidenciaron una diferencia estadísticamente significativa $(\mathrm{t}=-2.98, \mathrm{p}<.01)$ en el nivel promedio de estrés que reportan hombres $(\mathrm{M}=84.67)$ y mujeres $(\mathrm{M}=108.63)$. Cabe destacar que, aun cuando la media de ambos grupos recae en la categoría de "estrés intermedio", las mujeres reportan niveles de estrés significativamente más altos que los hombres.

El nivel de estrés laboral también se comparó en base a la organización jerárquica de la Institución. En este caso, la mayoría del personal obtuvo puntajes medios que los ubican en la categoría de estrés intermedio, pero cabe destacar que los docentes permanentes se ubicaron en el límite entre estrés intermedio y estrés alto, mientras que los mandos altos reportaron puntajes medios de estrés alto.

Asimismo, se compararon los puntajes medios en el nivel de estrés y las escalas de salud en función de la unidad administrativa a la que se pertenece. Los resultados se ilustran en las figuras 2,3 y 4 .

Figura 2

Puntuaciones medias en la escala de salud física en función de la unidad administrativa

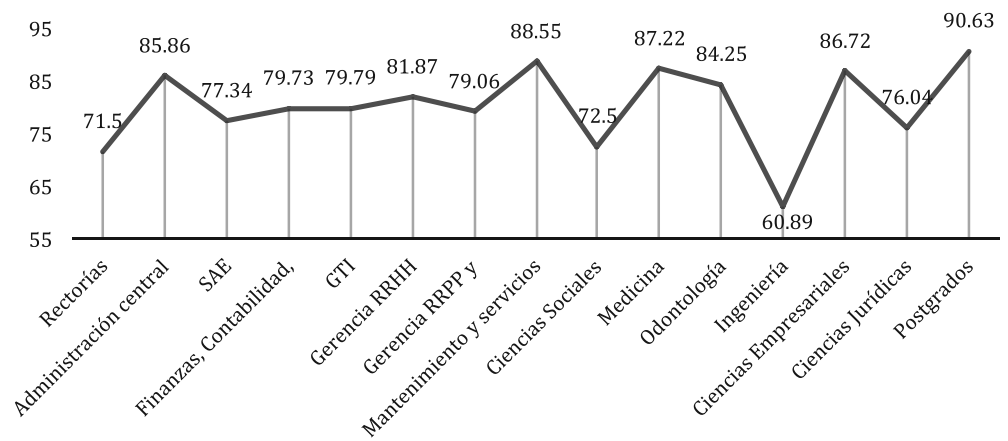




\section{Figura 3}

\section{Puntuaciones medias en la escala de salud mental en función de la unidad administrativa}

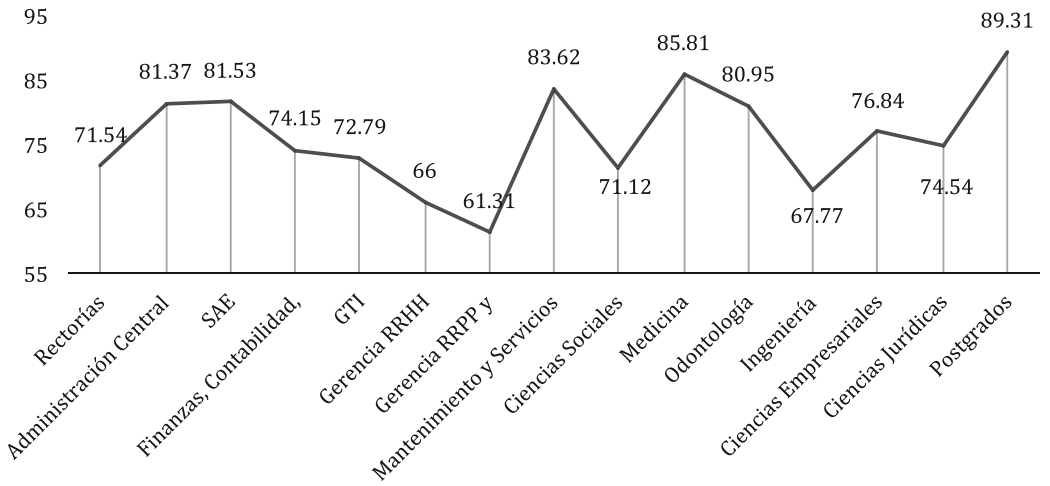

\section{Figura 4}

\section{Puntuaciones medias en el nivel de estrés laboral en función} de la unidad administrativa

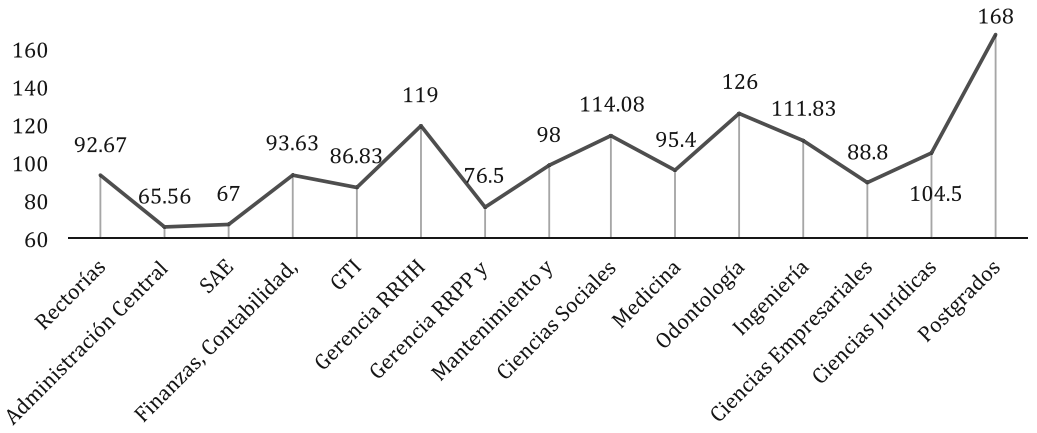

Finalmente, se solicitó a cada participante que brindara sugerencias para disminuir el estrés y favorecer la calidad de vida del personal de la Institución. Las respuestas se clasificaron en diferentes categorías, las cuales pueden observarse en la tabla 3 .

Tabla 3

Sugerencias para la disminución del estrés y mejora de la calidad de vida en la organización.

\begin{tabular}{|cccc|}
\hline Aspecto a mejorar & Frecuencia & Aspecto a mejorar & Frecuencia \\
\hline Salario & 58 & Limpieza & 5 \\
\hline Ambiente laboral & 52 & Mejora de parqueos & 5 \\
\hline Mobiliario y materiales de trabajo & 47 & Horario de trabajo & 4 \\
\hline Reconocimiento y motivación & 37 & Seguridad & 3 \\
\hline Relaciones interpersonales & 27 & Metas & 3 \\
\hline Capacitaciones & 23 & Acoso laboral & 3 \\
\hline Seguro médico y salud & 16 & Plazas laborales & 2 \\
\hline Trabajo en equipo & 11 & Mejora de cancha & 1 \\
\hline Religiosidad & 9 & Medio ambiente & 1 \\
\hline Transporte & 9 & & \\
\hline
\end{tabular}




\section{DISCUSIÓN}

A partir de los resultados obtenidos a través de la realización de este estudio se pueden generar diversas reflexiones sobre los datos encontrados. Un primer punto sobre el cual tratar es que, al igual que en investigaciones realizadas por otros autores, se ha encontrado que existe una relación entre el estrés laboral y la calidad de vida asociada a la salud, en donde altos niveles de estrés se relacionan con una calidad de vida disminuida y viceversa.

Esta relación también se manifiesta en los componentes de salud física y de salud mental de las personas, evidenciando la posibilidad de que el estrés sea un factor influyente en la generación de enfermedades físicas (Von Hopffgarten, 2013; Bauer-Delto, 2013) y desórdenes mentales (Mossop, 2013).

En relación específicamente a la calidad de vida asociada a la salud, si bien los puntajes medios de salud física y salud mental del personal de la Institución están encima de los promedios del grupo de referencia, vale la aclaración que esto no quiere decir que la organización esté exenta de problemáticas de salud, ni mucho menos que no existan factores que puedan influirla de manera negativa.

Entre estos factores cabe destacar el hecho de que buena parte de los participantes reportaron o bien laborar fuera del horario regular de trabajo, o bien llevar trabajo al hogar. Esto llama la atención, pues si la jornada laboral dura entre 8 y 10 horas (dependiendo del puesto de trabajo), parecería ser un periodo de tiempo suficiente para que el empleado cumpla con sus obligaciones; sin embargo, al menos para la tercera parte de los participantes, esto no es así. Ciertamente este tipo de prácticas podrían llevar a deterioros de salud, desgaste, descuido de las demás dimensiones de la vida, bajo desempeño, ausencias, entre otros (Díaz, 2011; Durán, 2010), por lo que es una situación nociva y poco deseable tanto desde el punto de vista organizacional como clínico.

Continuando con las reflexiones en torno a la calidad de vida asociada a la salud, un dato importante de destacar es la diferencia encontrada entre hombres y mujeres, donde se reflejó un menor puntaje de salud física, mental y general por parte de éstas. Esta situación podría explicarse desde el marco de referencia del machismo imperante en nuestra sociedad y la inequidad de género. Un primer aspecto es el fenómeno conocido como la "doble jornada laboral de la mujer", que implica dos jornadas que se encuentran constituidas por condiciones diferentes. La primera se le denomina "pública", y se encuentra caracterizada por relaciones laborales contractuales, con limites definidos en cuanto a contenido, carga, productividad, espacio, tiempo de trabajo, derechos y obligaciones; así como roles claros y fijos, y por personajes que concretan el lugar en las relaciones y en el trabajo mismo: ya sea patrón, administradores, etc. Y la segunda jornada, "privada", la doméstica, no considerada en sí como un trabajo, sino como parte de la naturaleza femenina (Programa de las Naciones Unidas para el Desarrollo 'PNUD', 2004).

Lógicamente, esta inequidad en la distribución de las labores entre hombres y mujeres supone una sobrecarga de trabajo de estas últimas, lo que las coloca en una posición desventajosa para el mantenimiento de un buen estado de salud física y mental.

Por otra parte, el machismo también fomenta conductas en los hombres que podrían explicar las diferencias entre los sexos citadas en los párrafos previos. Las 
creencias machistas de que los hombres son "fuertes", "no lloran", "deben tolerar el dolor", genera frecuentemente que éstos tiendan a percibirse con mejor estado de salud del que realmente tienen, o incluso a negar el padecimiento de alguna problemática, ya que esto implicaría un signo de "debilidad", incompatible con el ideal de "macho" imperante en nuestra sociedad (Gaborit, Rodríguez, Santori y Paz, 2003).

Siguiendo la línea reflexiva, en lo que respecta al estrés laboral es relevante destacar que, de acuerdo a los datos obtenidos, el promedio de la Institución recae en la categoría de un estrés intermedio. Dado que el instrumento utilizado para medir esta variable evalúa el estrés negativo (distrés), el nivel manifestado de este elemento refleja cierta nocividad para las personas.

En este sentido, el personal que se ubica en la categoría de "estrés bajo" son individuos que no manifiestan mayores consecuencias negativas a raíz del distrés percibido en el trabajo. En contraste, los participantes que se ubicaron en la categoría de "estrés alto" conforman la población que estaría siendo impactada de manera perjudicial y corren el riesgo más alto de desarrollar las problemáticas de salud que se asocian al estrés (Díaz, 2011; Durán, 2010). Estas personas requerirían de intervenciones que reviertan los efectos nocivos vivenciados. Por último, los individuos que se ubican en la categoría de "estrés intermedio" constituyen una población en riesgo de engrosar las filas de la categoría de "alto estrés", si no se pone en práctica un enfoque preventivo que evite esta transición.

Continuando con esta línea argumental, es relevante enfatizar que de las cinco posiciones jerárquicas que existen en la Universidad, sólo los "altos mandos" y los "docentes permanentes" reflejaron niveles altos de estrés. En el primer caso, esto se podría explicar debido a las exigencias propias del puesto que las personas desempeñan. Ocupar un cargo alto en la jerarquía organizacional puede implicar sobrecarga de trabajo, preocupaciones asociadas a la alta responsabilidad que el puesto conlleva (sobre todo si no se percibe apoyo social de parte del resto de actores sociales de la organización), alta demanda de tiempo que trae como consecuencia reducir los periodos dedicados a la familia, los hobbies, el descanso, entre otros.

En el segundo caso, el alto nivel de estrés reportado puede explicarse parcialmente por los argumentos mencionados en el párrafo anterior, sin embargo, hay que añadir el factor de sobrecarga que implica el desempeñar una profesión de servicio social. El ejercicio docente exige de forma inherente interpretar un rol de "cuidador", pues muchas veces se debe gestionar y lidiar con los distintos problemas emocionales que aquejan a los estudiantes. Es por esto que estos profesionales tienen el mayor riesgo de padecer el Síndrome de Burnout (Herranz-Bellido, Reig-Ferrer y Cabrero-García, 2006). Cabe destacar que el riesgo aumenta si la docencia se combina con otras profesiones que impliquen el servicio a los demás, tales como Medicina, Odontología, Psicología, Educación Especial, entre otros, pues la investigación sugiere que aquellos que fungen como "cuidadores" tienden a dejar de lado el cuido de sí mismos.

Como ya se ha mencionado, las dos variables que se han estudiado en este trabajo tienen un componente subjetivo muy importante; por esto, se consideró pertinente consultar a los participantes qué medidas consideran necesarias de implementar para reducir el estrés laboral y contribuir a una buena calidad de vida 
asociada a la salud en la Institución. Si bien se mencionaron acciones que se clasificaron en 19 categorías diferentes, se analizarán brevemente aquellas que fueron citadas con mayor frecuencia por los encuestados.

En primer lugar, la sugerencia que se realizó con mayor frecuencia es la mejora en salario del personal. Este es un elemento de gran importancia para la satisfacción, la motivación y las oportunidades de realización del empleado (Chiavenato, 2009), por lo que asegurar que éste se encuentre acorde al puesto de trabajo desempeñado, las exigencias laborales, la preparación del empleado, y sea competitivo a nivel de mercado es fundamental para favorecer la satisfacción dentro de la organización.

En esta misma línea se encuentran los reconocimientos, recompensas al buen trabajo y prestaciones que se brindan al personal. En este punto cabe enfatizar que las empresas con mayor éxito y con mayor satisfacción por parte de los empleados son aquellas que ofrecen mayor cantidad de privilegios y prestaciones a sus trabajadores (Smith, 2014).

Otro aspecto que se debe mejorar, según lo reportado por los empleados, es la calidad de los recursos materiales, tecnológicos y de mobiliario para realizar las diferentes tareas del trabajo. Este aspecto es muy relevante ya que tener los materiales adecuados se traduce en una mayor eficiencia en el desempeño laboral, mejor ergonomía y reducción de la probabilidad de ocurrencia de accidentes (Chiavenato, 2009).

Finalmente, tomando en cuenta que el personal contratado a tiempo completo pasa la mayor parte del día en la Institución, es imprescindible favorecer buenas relaciones interpersonales entre los trabajadores, tal como éstos lo han sugerido. Existe amplia evidencia que las relaciones sociales saludables y formar parte de redes de apoyo social son factores fundamentales para fomentar una buena calidad de vida, afrontar de manera más efectiva las dificultades y desarrollar resiliencia en los individuos (Polleti y Dobbs, 2002; Sarason y Sarason, 2006).

\section{CONCLUSIONES}

1. El estrés laboral y la calidad de vida asociada a la salud, tanto física como mental, son variables que están relacionadas de forma moderada en una dirección inversamente proporcional. Por lo tanto, esta evidencia apoya a la hipótesis general de la presente investigación.

2. Los empleados contratados a tiempo completo en la Universidad reportan experimentar, en promedio, un nivel de distrés intermedio. No obstante, esta evidencia no apoya a la hipótesis específica de investigación, pues se hipotetizó que, en promedio, los participantes reportarían un nivel de estrés laboral alto.

3. Los empleados contratados a tiempo completo en la Institución reflejaron un nivel de calidad de vida asociada a la salud, tanto física como mental, superior al grupo de comparación (muestra mexicana). Estos datos no apoyan a la hipótesis específica de investigación que sugería lo contrario.

4. El sexo femenino presentó una desventaja significativa en cuanto a la calidad de vida asociada a la salud física y mental, y niveles de estrés más altos en comparación a sus contrapartes masculinas. Este fenómeno podría deberse a factores relacionados con la inequidad de género y la ideología 
machista de la sociedad salvadoreña, sin embargo, se requiere mayor investigación para comprender con más exactitud este fenómeno.

5. Las personas que ejercen los cargos de "mandos altos" y "docentes permanentes" son las que reflejan mayores niveles de estrés. Esto puede deberse a diversos factores organizacionales relacionados con estos puestos. Cabe destacar que los docentes permanentes son especialmente vulnerables de padecer el Síndrome de Burnout debido a los aspectos emocionales intrínsecos a esta profesión. Dicho riesgo aumenta cuando la docencia se ejerce paralelamente con profesiones que implican servicio social.

6. Los participantes sugieren una gran cantidad de gestiones a realizar en orden a mejorar la calidad de vida dentro de la organización. Si bien es importante prestar atención a todas éstas, es fundamental actuar sobre aquellas en las que hay mayor consenso sobre su relevancia.

\section{RECOMENDACIONES}

\section{Académicas}

Realizar estudios de carácter cualitativo para conocer a profundidad las necesidades y las experiencias subjetivas del personal de la organización respecto a cómo impacta el trabajo en diferentes áreas de su vida. Estos datos servirían de complemento a la información cuantitativa recopilada en el presente informe.

Investigar los niveles de estrés laboral y calidad de vida asociada a la salud en el personal contratado a tiempo parcial y que labora fuera del campus central de la Universidad, ya que las dinámicas y procesos a los que estos individuos están sujetos podrían ser significativamente diferentes a los vivenciados por el personal contratado a tiempo completo.

Explorar de manera más específica las experiencias subjetivas de hombres y mujeres dentro de la organización, a fin de comprender con mayor precisión las diferencias entre ambos sexos encontradas en el presente estudio, y sus implicaciones.

Realizar estudios específicos en las diferentes unidades administrativas con el propósito de conocer detalladamente las dinámicas, procesos y necesidades de cada una de ellas, a fin de potenciar sus fortalezas y solventar sus problemáticas particulares, prestando especial atención a aquellas unidades que reflejaron un mayor nivel de afectación en salud física (Facultad de Ingeniería), salud mental (Facultad de Ingeniería, Gerencia de Relaciones Públicas y Comunicaciones, Gerencia de Recursos Humanos), y alto estrés (Escuela de Postgrados, Facultad de Odontología y Gerencia de Recursos Humanos).

\section{Metodológicas}

En futuras investigaciones, se recomienda mejorar la logística y la comunicación con las autoridades encargadas de los procesos investigativos, así como con los jefes de las diferentes unidades administrativas, con el propósito de evitar contratiempos y facilitar la participación de la totalidad del personal de la Universidad, aspecto que no se logró en el presente estudio.

\section{Intervención}

1. Implementar programas de psicoeducación sobre el estrés, sus 
características, consecuencias y formas de gestionarlo adecuadamente, tanto a través de técnicas cognitivas (gestión de tiempo, prácticas de autocuido, reestructuración cognitiva) así como también técnicas corporales (relajación, control de la respiración, meditación).

2. Desarrollar talleres de inteligencia emocional que permitan al personal de la Institución desarrollar habilidades de percepción y expresión emocional, comprensión de las propias emociones y sus efectos, así como también destrezas en el control y utilización de los procesos afectivos para adaptarse mejor al medio, tomar decisiones más acertadas respecto a sí mismo y los demás, y sostener relaciones interpersonales saludables y satisfactorias.

3. Fomentar las relaciones interpersonales saludables a través de la construcción de espacios de interacción donde el personal pueda compartir en dimensiones distintas al ámbito laboral, con el fin de desarrollar lazos de amistad, compañerismo y redes de apoyo social, tanto a un nivel microsocial (cohesión dentro de cada unidad administrativa) como macrosocial (cohesión de la organización en su totalidad).

\section{FUENTES CONSULTADAS}

Alonso, J., Prieto, L. y Antó, M. (1995). “La versión española del SF-36 Health Survey (Cuestionario de Salud SF-36): un instrumento para la medida de los resultados clínicos”. Medicina Clínica, 104 (20), 771-776.

Bauer-Delto, A. (2013). "Nervios a flor de piel”. Mente y Cerebro, 59, 57-59.

Chiavenato, I. (2009). Gestión del talento humano (3a ed.). México D.F.: McGraw-Hill.

Díaz, D. (2011). “Estrés laboral y sus factores de riesgo psicosocial”. Revista CES Salud Pública, 2 (1), 80-84.
Durán, M. (2010). "Bienestar psicológico: el estrés y la calidad de vida en el contexto laboral". Revista Nacional de Administración, 1 (1), 71-84.

Durán, L., Gallegos, K. y Salinas, G. (2004). “Hacia una base normativa mexicana en la medición de la calidad de vida relacionada con la salud, mediante el Formato Corto 36". Salud Pública de México, 46 (4), 306-315.

Feldman, R. (2010). Psicología con aplicaciones en países de habla hispana (8 ${ }^{\mathrm{a}}$ ed.). México D.F.: McGraw-Hill.

Gaborit, M., Rodríguez, M., Santori, A. y Paz, C. (2003). Más allá de la invisibilidad: disparidad de género en El Salvador. San Salvador: UCA Editores.

García-Portilla, M., Bascarán, M., Sáiz, P., Bousoño, M. y Bobes, J. (2006). Banco de instrumentos básicos

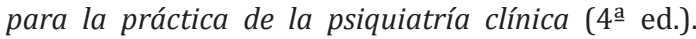
Buenos Aires: Ars Medica.

Hernández, R., Fernández, C. y Baptista, M. (2010). Metodología de la investigación (5a ed.). México, D.F.: McGraw-Hill.

Herranz-Bellido, J., Reig-Ferrer, A. y Cabrero-García, J. (2006). "La prevalencia del estrés laboral asistencial entre los profesores universitarios". Análisis y Modificación de Conducta, 32 (146), 743-766.

Marrero, M., Rivero, E., Pastor, M., Fernández, C y Vergara, A. (2011). “Elaboración de la versión cubana del cuestionario JSS para la evaluación del estrés psicosocial laboral". Revista Cubana de Salud y Trabajo, 12 (2), 9-18.

Morrison, V. y Bennett, P. (2008). Psicología de la salud. Madrid: Pearson Prentice Hall.

Mossop, B. (2013). "Sobrecarga en el cerebro". Mente y Cerebro, 59, 62-65.

Peiró, J. y Rodríguez, I. (2008). "Estrés laboral, liderazgo y salud organizacional". Papeles del Psicólogo, 29 (1), 68-82.

Polleti, R. y Dobbs, B. (2002). La resiliencia, el arte de resurgir a la vida. Buenos Aires: Lumen.

Programa de las Naciones Unidas para el Desarrollo. (2004). Equidad de Género de El Salvador. Cuadernos sobre desarrollo humano: género. San Salvador.

Rosenzweig, M. y Leiman, A. (1992). Psicología Fisiológica (2 ${ }^{\mathrm{a}}$ ed.). Madrid: McGraw-Hill.

Sarason, I. y Sarason, B. (2006). Psicopatología. Psicología anormal: el problema de la conducta inadaptada (11a ed.). México: Pearson Educación. 
Smith, J. (2014). "The Best Companies to Work for in 2014". Forbes. Disponible en URL: http://www. forbes.com/sites/jacquelynsmith/2013/12/11/ the-best-companies-to-work-for-in-2014

Von Hopffgarten, A. (2013). "Protección mental". Mente y Cerebro, 59, 50-56.
Zúniga, M., Carrillo-Jiménez, G., Fos, P., Gandek, B. y Medina-Moreno, M. (1999). "Evaluación del estado de salud con la Encuesta SF-36: resultados preliminares en México". Salud Pública de México, 41 (2), 110-118. 